\title{
Search for Neutrinos in Super-Kamiokande Associated with Gravitational Wave Events ${ }^{\dagger}$
}

\author{
Yuuki Nakano $\ddagger(1)$ and On behalf of the Super-Kamiokande Collaboration $\ddagger, \S$ \\ Kamioka Observatory, Institute for Cosmic Ray Research, The University of Tokyo, Higashi-Mozumi 456, \\ Kamioka-cho, Hida-city Gifu 506-1205, Japan; ynakano@km.icrr.u-tokyo.ac.jp; Tel.: +81-578-85-9665 \\ + This paper is based on the talk at the 7th International Conference on New Frontiers in Physics (ICNFP 2018), \\ Crete, Greece, 4-12 July 2018. \\ $\ddagger$ These authors contributed equally to this work. \\ $\S$ Membership of the Super-Kamiokande Collaboration is provided in the Acknowledgments.
}

Received: 30 November 2018; Accepted: 26 December 2018; Published: 29 December 2018

check for updates

\begin{abstract}
We report the results from a search in Super-Kamiokande for neutrino signals coincident with gravitational-wave events using a neutrino energy range from 3.5 MeV-100 PeV. We searched for coincident neutrino events within a time window of $\pm 500 \mathrm{~s}$ around the gravitational-wave detection time. In this work, we report the number of events within the search-window and the $90 \%$ confidence level upper limits on the neutrino fluence for each gravitational-wave event.
\end{abstract}

Keywords: Super-Kamiokande; neutrino; gravitational wave; multi-messenger; GW150914; GW151226; GW170817

\section{Introduction}

\subsection{Era of Multi-Messenger Astronomy}

After the discovery of the gravitational wave from the black hole (BH) merger of GW150914 [1,2] by Advanced LIGO [3], the era of multi-messenger astronomy has started. The goals of multi-messenger observations are to understand an evolution of cosmic processes through a combination of observed signals. For this purpose, many efforts to search for coincidences in the observational data in complementary experiments have been made.

Since then, other BH-BH mergers (GW151226 [4], GW170104 [5], GW170814 [6], GW170608 [7]), as well as a neutron star (NS) merger (GW170817 [8]) have been observed by both Advanced LIGO and Advanced Virgo [9]. Currently, eleven GW events in total have been reported (including GW151012, GW170729, GW170809, GW170818, and GW170823) [10]. In the case of GW170817, the first joint detection of gravitational waves and electromagnetic radiation from a single source has been performed [11,12]. Soon after the observation of GW170817, a short gamma-ray burst (GRB170817A) that followed the merger of this binary was also detected by the Fermi GBM [13] and INTEGRAL [14]. In addition, many observations have been reported by X-ray $[15,16]$ and radio bands $[17,18]$, while no neutrino signal [19] consistent with the source has been found in follow-up searches.

\subsection{Neutrino Detectors}

Neutrinos from a binary merger (BH-BH or NS-NS) can open a new window for multi-messenger research since a mechanism for the neutrino emission from such mergers is unclear. Currently, several neutrino detectors have accumulated neutrino signals with various techniques. They can search for neutrino signals at any time around a gravitational event because they continuously take data observing the whole sky. 
Several searches have been performed for the neutrino counterparts of either binary BH-BH merges or the binary NS-NS merger [19-28], as summarized in Table 1. However, no significant neutrino signal has been observed by the neutrino telescopes, so far.

Table 1. Summary of neutrino detectors and references for their searches.

\begin{tabular}{ccc}
\hline Experiment & Typical Energy Range & Reference \\
\hline Borexino & $250 \mathrm{keV}-15 \mathrm{MeV}$ & {$[20]$} \\
KamLAND & $1.8 \mathrm{MeV}-111 \mathrm{MeV}$ & {$[21]$} \\
Super-Kamiokande (SK) & $3.5 \mathrm{MeV}-100 \mathrm{PeV}$ & {$[22,23]$} \\
Baikal-GVD & $5 \mathrm{TeV}-10 \mathrm{PeV}$ & {$[24]$} \\
ANTARES & $100 \mathrm{GeV}-100 \mathrm{TeV}$ & {$[19,25-27]$} \\
IceCube & $100 \mathrm{GeV}-100 \mathrm{TeV}$ & {$[19,25-27]$} \\
Peirre Auger & $100 \mathrm{PeV}-25 \mathrm{EeV}$ & {$[19,28]$} \\
\hline
\end{tabular}

In this work, we report the results of a search in the Super-Kamiokande (SK) detector for neutrino signals associated with the gravitational events based on [22,23].

\section{Super-Kamiokande Detector and Analysis Method}

\subsection{Detector}

The Super-Kamiokande (SK) is a water Cherenkov detector located at $1000 \mathrm{~m} \mathrm{(2700} \mathrm{m} \mathrm{water}$ equivalent) below the top of Mt. Ikenoyama in Gifu prefecture, Japan [29]. It is a cylindrical stainless tank structure and contains 50 ktons of ultra-pure water. The detector is divided into two regions of its structure to separate them optically with a Tyvek sheet [30]; one is an inner detector (ID), and the other is an outer detector (OD). The ID serves as the target of interactions, and the OD is used to veto external cosmic ray muons. In the ID detector, 11,129 20-inch photomultipliers (PMTs) [31] are placed to observe the Cherenkov light produced by penetrating charged particles. On the other hand, 1885 8-inch PMTs are placed to detect cosmic ray muons. The configuration of the detector and its performance are described $[29,30]$.

The first phase of the SK experiment (SK-I) began with the original configuration in April 1996. The later phases are described in Table 2. In May 2018, the forth phase of SK (SK-IV) ended the physics data taking to refurbish the detector for the next project of SK (SK-Gd).

Table 2. Experimental phases of the Super-Kamiokande detector. ID, inner detector; OD, outer detector; PMT, photomultiplier.

\begin{tabular}{ccccc}
\hline Phase & SK-I & SK-II & SK-III & SK-IV \\
\hline Period & April 1996-July 2001 & October 2002-October 2005 & July 2006-August 2008 & September 2008-May 2018 \\
ID PMTs [31] & 11,149 & 5182 & 11,129 & 11,129 \\
OD PMTs & 1885 & 791 & 1885 & 1885 \\
Photo coverage & $40 \%$ & $19 \%$ & $40 \%$ & $40 \%$ \\
Electronics & ATM [32,33] & ATM & ATM & Qbee [34,35] \\
\hline
\end{tabular}

The ring pattern of the observed Cherenkov light produced by charged particles enables the detector to reconstruct the initial interaction vertex and the energy. With this water Cherenkov technique, SK can study many interactions between neutrinos and nuclei, as well as proton decay in the energy range from a few $\mathrm{MeV}$ to tens of $\mathrm{GeV}$ inside the detector.

\subsection{Analysis to Search for Neutrino Candidates}

SK has sensitivity to a wide range of neutrino energies from a few $\mathrm{MeV}$ to $\sim 100 \mathrm{PeV}$. Neutrino events below $100 \mathrm{MeV}$ in SK are categorized as the "low energy sample" and typically used to study solar neutrinos [36] and to search for core-collapse supernova neutrinos [37]. In this energy range, 
radioactive impurities [38], spallation products from cosmic ray muons [39], atmospheric and solar neutrinos are the main background for the coincidence search. Above $100 \mathrm{MeV}$, neutrino events are categorized as "high energy samples" and typically used to study atmospheric neutrinos [40] and to search for proton decays. This sample consists of three distinct topologies [41]: fully-contained (FC), partially-contained (PC), and upward-going muon (UPMU). In this energy range, only atmospheric neutrinos are the main background. The details of these analyses can be found in [36,37,40,41].

In order to search for a neutrino candidate event, we selected a search window of $\pm 500 \mathrm{~s}$ around the detection time of each gravitational wave event. The expected background rate within this search window, as well as the typical energy range of each sample are summarized in Table 3.

Table 3. Number of expected background events within a search window of $1000 \mathrm{~s}$ for all samples. In order to estimate the rate, we used 306.1 days of SK data for the solar sample, 2887 days for the relic sample, and 2976 days for the fully-contained (FC), partially-contained (PC), and upward-going muon (UPMU) samples [23].

\begin{tabular}{ccc}
\hline Sample Name & Typical Energy Range & Expected Background Rate (Event/(1000 s)) \\
\hline Solar & $3.5-15.5 \mathrm{MeV}$ & $2.90 \pm 0.01$ \\
Relic & $15.5-100.0 \mathrm{MeV}$ & $(1.93 \pm 0.08) \times 10^{-3}$ \\
FC & $100 \mathrm{MeV}-10 \mathrm{GeV}$ & $(9.36 \pm 0.06) \times 10^{-2}$ \\
PC & $100 \mathrm{MeV}-10 \mathrm{GeV}$ & $(7.52 \pm 0.23) \times 10^{-3}$ \\
UPMU & $1.6 \mathrm{GeV}-100 \mathrm{PeV}$ & $(1.64 \pm 0.02) \times 10^{-2}$ \\
\hline
\end{tabular}

\section{Neutrinos from Black Hole-Black Hole Mergers}

\subsection{Introduction}

Searching for an electromagnetic counterpart is critically important for a gravitational wave event. In the case of GW150914, many searches for coincidence signals have been performed around the detection time of GW150914. Eventually, no astronomical counterpart with a BH-BH merger event has been observed except for a weak coincident excess reported by Fermi GBM of about $0.4 \mathrm{~s}$ after GW150914 [42].

In general, there is no theory of neutrino generation associated with a $\mathrm{BH}-\mathrm{BH}$ merger. However, some possibilities of high energy neutrino emission have been discussed; for example, relativistic jets when an accretion disk is formed around the source $[43,44]$, or the synthesis of heavy elements with the accretion disk [45,46]. A search for neutrinos from $\mathrm{BH}-\mathrm{BH}$ mergers gives the opportunity to obtain a more complete picture of emission processes.

\subsection{Search for Neutrinos around GW150914/GW151226}

We have carried out the search for neutrinos from both GW150914 and GW151226. After the reduction cuts, four events were found in the solar sample, while no event was found in either the atmospheric or relic samples within $\pm 500 \mathrm{~s}$ around GW150914. Figure 1a shows the energy distribution as a function of time, and Figure $1 \mathrm{~b}$ shows the energy distribution of remaining events together with the expected background spectrum. According to the background rate for the solar sample described in Table 3, the probability of finding four or more events passing the reduction cuts is calculated to be $33.0 \%$. 


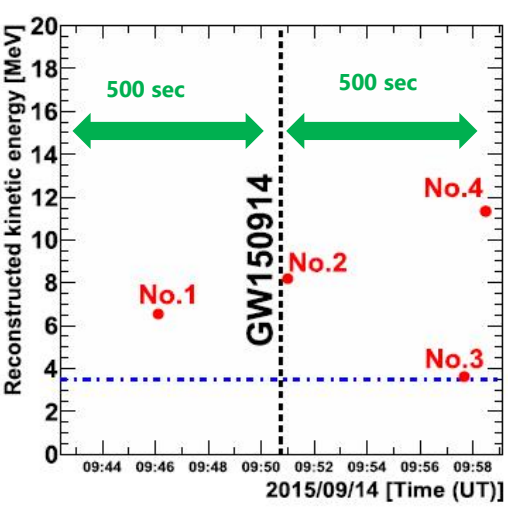

(a)

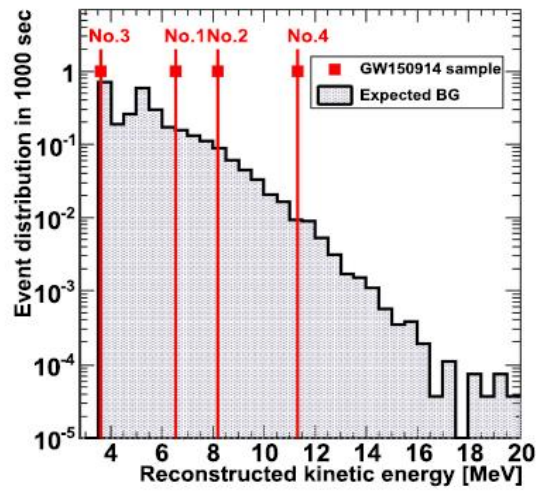

(b)

Figure 1. (a) The timing distribution within the \pm 500 -s time window around GW150914. (b) The energy distribution of the observed events (red points) within $\pm 500 \mathrm{~s}$ together with the expected background (gray histogram). The original figures were adopted from [22].

Figure 2 shows the sky map using the reconstructed direction of the charged particle in the remaining four events, as well as the 90\% C.L. contour for the location of GW150914 [1]. In this analysis, we assumed all interaction occurred via electron-neutrino elastic scattering because of its strong correlation with the incident neutrino direction (very weak anti-correlation exists in the case of the inverse beta decay [47]). The angular resolution of the charged particle below $18.0 \mathrm{MeV}$ is calibrated by the electron LINAC [48].



Figure 2. Reconstructed directions of the charged particle in the remaining four events associated with GW150914. The red region is the 90\% C.L. contour for the location of GW150914 by LIGO [1]. The black points are the remaining events together with their angular resolution (gray region). The cross is the location of the Sun at the detection time. The original figure was adopted from [22].

For GW151226 [4], we did not find any event within $\pm 500 \mathrm{~s}$ after the reduction cuts in any sample. The probability of finding no event passing the reduction cuts is $5.5 \%$.

\subsection{Neutrino Fluence Limit}

The number of neutrino candidate events observed in the search window can be converted into an upper limit on neutrino fluence. In this analysis, the method developed in $[49,50]$ was used. For the calculation, the $90 \%$ C.L. limit on the number of neutrino events in the search window calculated using a Poisson distribution with a background, the number of events after the reduction cuts, the number of target nuclei in the detector, the total cross-section for all interaction based on NEUT 5.3.5 [51], the reduction efficiencies for each analysis sample and the detector response were considered. For both solar and relic samples, we used the energy spectrum with an index of zero since there was no reason to assume a power spectrum of neutrino emission in this energy region. For FC/PC/UPMU samples, 
we used the energy spectrum with an index of -2 because this index is commonly assumed for astrophysical neutrinos accelerated by shocks [52]. Only for the UPMU sample, the shadowing effect of neutrinos due to the Earth, as well as the dependence of the zenith angle of incoming neutrinos were additionally considered.

Table 4 summarizes the $90 \%$ C.L. limit on neutrino fluence obtained in this analysis. The obtained results gave the most stringent limits for neutrino emission in the energy range between $\sim 10 \mathrm{MeV}$ and $100 \mathrm{GeV}$ (a comparison of fluence limits below $100 \mathrm{MeV}$ was discussed in [53]).

Table 4. Neutrino fluence limits at 90\% C.L. from GW150914, GW151226, and GW170817. The unit for the neutrino fluence is $\mathrm{cm}^{-2}$. For GW170817 (NS-NS merger), two energy spectrum of neutrino emission is used; one is the flat spectrum with an index of zero, and the other is the Fermi-Dirac distribution with an average energy of $20 \mathrm{MeV}$. The latter is described in round brackets.

\begin{tabular}{cccc}
\hline Neutrino (Sample) & GW150914 & GW151226 & GW170817 \\
\hline$v_{\mu}(\mathrm{FC}+\mathrm{P} \mathrm{C})$ & $5.6 \times 10^{4}$ & $5.6 \times 10^{4}$ & $5.6 \times 10^{4}$ \\
$\bar{v}_{\mu}(\mathrm{FC}+\mathrm{PC})$ & $1.3 \times 10^{4}$ & $1.3 \times 10^{4}$ & $1.3 \times 10^{4}$ \\
$v_{e}(\mathrm{FC}+\mathrm{PC})$ & $4.8 \times 10^{4}$ & $4.8 \times 10^{4}$ & $4.8 \times 10^{4}$ \\
$\bar{v}_{e}(\mathrm{FC}+\mathrm{PC})$ & $1.2 \times 10^{4}$ & $1.2 \times 10^{4}$ & $1.2 \times 10^{4}$ \\
\hline$v_{\mu}(\mathrm{UPMU})$ & $14-37$ & $14-37$ & $16.0_{-0.6}^{+0.7}$ \\
$\bar{v}_{\mu}$ (UPMU) & $19-50$ & $19-50$ & $21.3_{-0.8}^{+1.1}$ \\
\hline$v_{e}($ solar + relic) & $3.0 \times 10^{9}$ & $1.3 \times 10^{9}$ & $1.0 \times 10^{9}\left(3.4 \times 10^{9}\right)$ \\
$\bar{v}_{e}$ (solar + relic) & $4.2 \times 10^{7}$ & $1.8 \times 10^{7}$ & $1.2 \times 10^{7}\left(6.6 \times 10^{7}\right)$ \\
$v_{x}$ (solar + relic) & $1.9 \times 10^{10}$ & $8.1 \times 10^{9}$ & $6.3 \times 10^{9}\left(2.1 \times 10^{10}\right)$ \\
\hline
\end{tabular}

\section{Neutrinos from a Neutron Star Merger}

\subsection{Introduction}

The electromagnetic counterparts for binaries of NS-NS or NS-BH have actively been discussed; for example, short gamma-ray burst [45,54], kilonova/micronova [55], and so on. Some theoretical predictions of neutrino emission via an NS merger have been proposed. A similar mechanism as for core-collapse supernova can produce neutrinos whose average energy is typically a few tens of $\mathrm{MeV}$ based on the recent numerical simulations [56-58].

Since the two-week-long electromagnetic counterpart after GW170817 was observed [59], ANTARES, IceCube, the Pierre Auger Observatory, and Baikal-GVDhave searched for neutrino signals with two different time windows [19,24]; one is $\pm 500 \mathrm{~s}$ around the merger to search for neutrinos associated with prompt and extended gamma-ray emissions [60-62], and the other is 14 days after the merger to search for longer-lived emission processes [63].

\subsection{Search for Neutrinos within \pm 500 s around GW170817}

We searched for neutrinos within the window of $\pm 500 \mathrm{~s}$ and found no event in relic/FC/PC/UPMU samples. For the solar sample, we note that we have performed the calibration of LINAC [48] from 3-22 August 2017. Fortunately, the physics run was operated at the time of GW170817. However, many low-energy events occurring near the surface of the LINAC pipes have been observed because radioactive impurities existed on the pipes in the water tank. In order to remove such additional background events occurring on the surface of the pipe, we additionally applied the calibration source cut to the solar sample [36]. Ultimately, no event was found in the solar sample. The probability of no event after the reduction cut is $5.5 \%$. Using the null number for each sample, we obtained the neutrino fluence limits within $\pm 500 \mathrm{~s}$ around GW170817. The results are summarized in Table 4 . In addition to the energy spectrum of neutrino emission with an index of zero in the low energy sample, we also used another spectrum assuming a Fermi-Dirac distribution with an average energy of $20 \mathrm{MeV}$ since such models are well studied based on the simulation $[56,57]$. 


\subsection{The Following 14-Day Time Window}

In order to search for longer-lived emission process, we searched for neutrinos in the 14-day window after GW170817. The effective lifetime of the window for each sample and the observed event, as well as the expected background event are summarized in Table 5.

Table 5. Analysis results of the 14-day window search. Lifetime for each sample, the observed events within the lifetime, and the expected background event within the lifetime are described. As mentioned in the main text, we did not carry out the search in the solar sample due to the large amount of background due to radioactive impurities.

\begin{tabular}{cccc}
\hline Sample Name & Lifetime & Observed Event (Event/Lifetime) & Expected Event (Event/Lifetime) \\
\hline Solar & - & Many radioactive impurities & - \\
Relic & 9.15 & 2 & $1.53 \pm 0.06$ \\
FC & 11.30 & 76 & $91.44 \pm 0.57$ \\
PC & 11.30 & 8 & $7.35 \pm 0.23$ \\
UPMU & 11.30 & 0 & $16.05 \pm 0.23$ \\
UPMU $\left(\leq 5^{\circ}\right)$ & 11.30 & & $(6.11 \pm 0.04) \times 10^{-2}$ \\
\hline
\end{tabular}

As explained before, we observed many background events due to radioactive impurities in the water tank. Because of this, the quality of the event selection and reconstruction in the solar sample was not stable. Therefore, we did not use the solar sample in the 14-day time window. In the relic sample, we found two events on 24 August 2017 10:33:04 UTC and on 28 August 2017 14:36:34 UTC, where the expected background rate was $1.53 \pm 0.06$. The probability of observing two or more events is $45.1 \%$. In the FC/PC/PUMU samples, we found 76, 8, and 13 events within the 14-day time window, where their expected backgrounds were $91.44 \pm 0.57,7.35 \pm 0.23$, and $16.05 \pm 0.23$, respectively. Therefore, no excess over the background was observed in any sample.

Figure 3 shows the sky map of the observed event within the following 14-day time window. Since the UPMU events were higher in energy than the other topologies, their angular resolution was better. Therefore, the detected lepton pointed back to the incoming neutrino with more accuracy, allowing for a smaller search cone [64]. With this method, we concentrated on $\mathrm{a} \pm 5^{\circ}$ cone around the location of GW170817 and found no event in this cone in the 14-day time window.
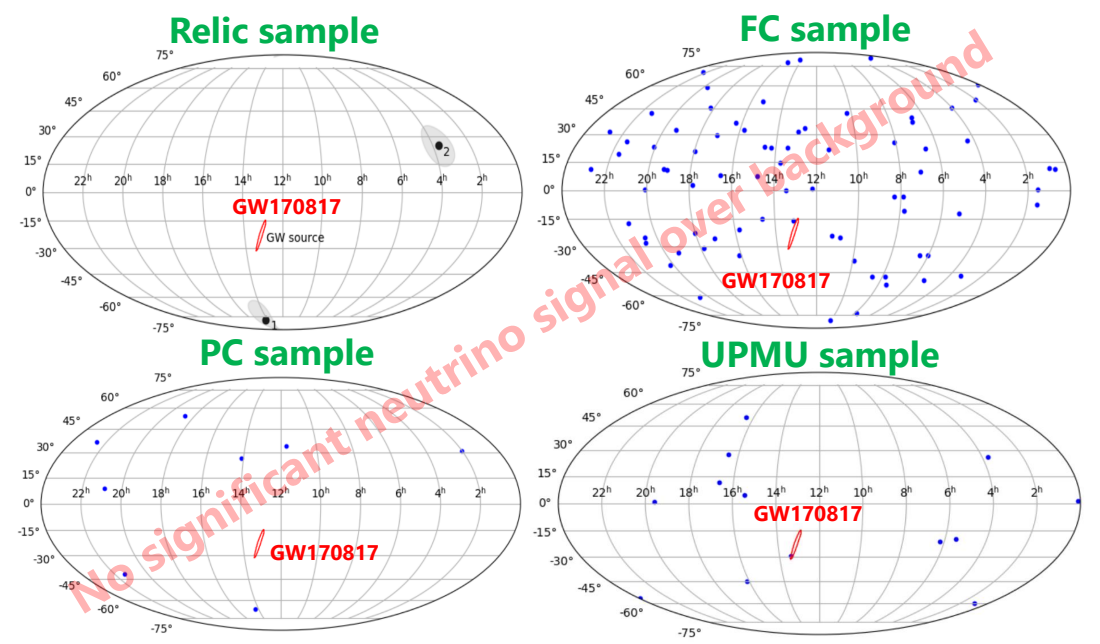

Figure 3. Sky map of the observed events in the 14-day time window. The points (red contour) show the observed events (location of GW170817). 


\section{Conclusions}

We carried out coincidence searches in SK for neutrino signals with gravitational waves GW150914, GW151226, and GW170817 using a wide energy range from 3.5 MeV-100 PeV. The analysis was performed within a time window of $\pm 500 \mathrm{~s}$, as well as 14 days after the merger. Four neutrino candidates were found within the $\pm 500 \mathrm{~s}$ time window for GW150914. These were consistent with the estimated background. For GW151226 and GW170817, no event was observed in the $\pm 500 \mathrm{~s}$ window. For the 14-day time window of GW170817, no significant neutrino signal was observed over the expected background. The $90 \%$ C.L. limits on neutrino fluence were determined considering the detector performance. As mentioned, more details about this study can be found in [22,23].

Author Contributions: These authors contributed equally to this work.

Funding: The Super-Kamiokande experiment has been built and operated from funding by the Japanese Ministry of Education, Culture, Sports, Science and Technology, the U.S. Department of Energy, and the U.S. National Science Foundation.

Acknowledgments: We gratefully acknowledge the cooperation of the Kamioka Mining and Smelting Company.

Conflicts of Interest: The authors declare no conflict of interest.

\section{References}

1. Abbott, B.P.; et al. [LIGO Scientific Collaboration and Virgo Collaboration]. Observation of Gravitational Waves from a Binary Black Hole Merger. Phys. Rev. Lett. 2016, 116, 061102. [CrossRef] [PubMed]

2. Abbott, B.P.; et al. [LIGO Scientific Collaboration and Virgo Collaboration]. Binary Black Hole Mergers in the First Advanced LIGO Observing Run. Phys. Rev. X 2016, 6, 041015.

3. Abadie, J.; et al. [The LIGO Scientific Collaboration]. Advanced LIGO. Class. Quantum Grav. 2015, 32, 074001.

4. Abbott, B.P.; et al. [LIGO Scientific Collaboration and Virgo Collaboration]. GW151226: Observation of Gravitational Waves from a 22-Solar-Mass Binary Black Hole Coalescence. Phys. Rev. Lett. 2016, 116, 241103. [CrossRef]

5. Abbott, B.P.; et al. [LIGO Scientific Collaboration and Virgo Collaboration]. GW170104: Observation of a 50-Solar-Mass Binary Black Hole Coalescence at Redshift 0.2. Phys. Rev. Lett. 2017, 118, 221101. [CrossRef] [PubMed]

6. Abbott, B.P.; et al. [LIGO Scientific Collaboration and Virgo Collaboration]. GW170814: A Three-Detector Observation of Gravitational Waves from a Binary Black Hole Coalescence. Phys. Rev. Lett. 2017, 119, 141101. [CrossRef] [PubMed]

7. Abbott, B.P.; et al. [LIGO Scientific Collaboration and Virgo Collaboration]. GW170608: Observation of a 19 Solar-mass Binary Black Hole Coalescence. Astrophys. J. Lett. 2017, 851, L35. [CrossRef]

8. Abbott, B.P.; et al. [LIGO Scientific Collaboration and Virgo Collaboration]. GW170817: Observation of Gravitational Waves from a Binary Neutron Star Inspiral. Phys. Rev. Lett. 2017, 119, 161101. [CrossRef]

9. Acernese, F.; Agathos, M.; Agatsuma, K.; Aisa, D.; Allemandou, N.; Allocca, A.; Amarni, J.; Astone, P.; Balestri, G.; Ballardin, G.; et al. Advanced Virgo: A second-generation interferometric gravitational wave detector. Class. Quantum Grav. 2015, 32, 024001. [CrossRef]

10. Abbott, B.P.; et al. [LIGO Scientific Collaboration]. A Gravitational-Wave Transient Catalog of Compact Binary Mergers Observed by LIGO and Virgo during the First and Second Observing Runs. arXiv 2018, arXiv:1811.12907.

11. Abbott, B.P.; Abbott, R.; Abbott, T.D.; Acernese, F.; Ackley, K.; Adams, C.; Adams, T.; Addesso, P.; Adhikari1, R.X.; Adya, V.B.; et al. Multi-messenger Observations of a Binary Neutron Star Merger*. Astrophys. J. Lett. 2017, 848, L12. [CrossRef]

12. Abbott, B.P.; Abbott, R.; Abbott, T.D.; Acernese, F.; Ackley, K.; Adams, C.; Adams, T.; Addesso, P.; Adhikari1, R.X.; Adya, V.B.; et al. Gravitational Waves and Gamma-Rays from a Binary Neutron Star Merger: GW170817 and GRB 170817A. Astrophys. J. Lett. 2017, 848, L13.

13. Goldstein, A.; Veres, P.; Burns, E.; Briggs, M.S.; Hamburg, R.; Kocevski, D.; Wilson-Hodge, C.A.; Preece, R.D.; Poolakkil, S.; Roberts, O.J.; et al. An Ordinary Short Gamma-Ray Burst with Extraordinary Implications: Fermi-GBM Detection of GRB 170817A. Astrophys. J. Lett. 2017, 848, L14. [CrossRef] 
14. Savchenko, V.; Ferrigno, C.; Kuulkers, E.; Bazzano, A.; Bozzo, E.; Brandt, S.; Chenevez, J.; Courvoisier, T.J.-L.; Diehl, R.; Domingo, A.; et al. INTEGRAL Detection of the First Prompt Gamma-Ray Signal Coincident with the Gravitational-wave Event GW170817. Astrophys. J. Lett. 2017, 848, L15. [CrossRef]

15. Troja, E.; Piro, L.; van Eerten, H.; Wollaeger, R.T.; Im, M.; Fox, O.D.; Butler, N.R.; Cenko, S.B.; Sakamoto, T.; Fryer, C.L.; et al. The X-ray counterpart to the gravitational-wave event GW170817. Nature 2017, 551, 71-74. [CrossRef]

16. Margutti, R.; Berger, E.; Fong, W.; Guidorzi, C.; Alexander, K.D.; Metzger, B.D.; Blanchard, P.K.; Cowperthwaite, P.S.; Chornock, R.; Eftekhari, T.; et al. The Electromagnetic Counterpart of the Binary Neutron Star Merger LIGO/Virgo GW170817. V. Rising X-Ray Emission from an Off-axis Jet. Astrophys. J. Lett. 2017, 848, L20.

17. Hallinan, G.; Corsi, A.; Mooley, K.P.; Hotokezaka, K.; Nakar, E.; Kasliwal, M.M.; Kaplan, D.L.;Frail, D.A.; Myers, S.T.; Murphy, T.; et al. A radio counterpart to a neutron star merger. Science 2017, 358, 1579-1583. [CrossRef] [PubMed]

18. Alexander, K.D.; Berger, E.; Fong, W.; Williams, P.K.G.; Guidorzi, C.; Margutti, R.; Metzger, B.D.; Annis, J.; Blanchard, P.K.; Brout, D.; et al. The Electromagnetic Counterpart of the Binary Neutron Star Merger LIGO/Virgo GW170817. VI. Radio Constraints on a Relativistic Jet and Predictions for Late-time Emission from the Kilonova Ejecta. Astrophys. J. Lett. 2017, 848, L21. [CrossRef]

19. Albert, A.; et al. [LIGO Scientific Collaboration and Virgo Collaboration]. Search for High-energy Neutrinos from Binary Neutron Star Merger GW170817 with ANTARES, IceCube, and the Pierre Auger Observatory. Astrophys. J. Lett. 2017, 850, L35. [CrossRef]

20. Albert, M.; et al. [The Borexino Collaboration]. A Search for Low-energy Neutrinos Correlated with Gravitational Wave Events GW 150914, GW 151226, and GW 170104 with the Borexino Detector. Astrophys. J. 2017, 850, L21.

21. Gando, A.; et al. [The KamLAND Collaboration]. A Search for Electron Antineutrinos Associated with Gravitational-wave Events GW150914 and GW151226 Using KamLAND. Astrophys. J. Lett. 2016, 829, L34. [CrossRef]

22. Abe, K.; et al. [Super-Kamiokande Collaboration]. Search for Neutrinos in Super-Kamiokande Associated with Gravitational-Wave Events GW150914 and GW151226. Astrophys. J. Lett. 2016, 830, L11. [CrossRef]

23. Abe, K.; et al. [Super-Kamiokande Collaboration]. Search for Neutrinos in Super-Kamiokande Associated with the GW170817 Neutron-star Merger. Astrophys. J. Lett. 2018, 857, L4. [CrossRef]

24. Avrorin, A.D.; et al. [Baikal-GVD Collaboration]. Search for high-energy neutrinos from GW170817 with Baikal-GVD neutrino telescope. arXiv 2018, arXiv:1810.10966.

25. Adrián-Martínez, S.; et al. [Antares Collaboration, IceCube Collaboration, LIGO Scientific Collaboration, and Virgo Collaboration]. High-energy neutrino follow-up search of gravitational wave event GW150914 with ANTARES and IceCube. Phys. Rev. D 2016, 93, 122010. [CrossRef]

26. Albert, A.; et al. [ANTARES Collaboration, IceCube Collaboration, LIGO Scientific Collaboration, and Virgo Collaboration]. Search for high-energy neutrinos from gravitational wave event GW151226 and candidate LVT151012 with ANTARES and IceCube. Phys. Rev. D 2017, 96, 022005. [CrossRef]

27. Albert, A.; et al. [ANTARES, IceCube, LIGO, Virgo Collaborations]. Search for Multi-messenger Sources of Gravitational Waves and High-energy Neutrinos with Advanced LIGO during its first Observing Run, ANTARES and IceCube. arXiv. 2018, arXiv:1810.10693.

28. Aad, A.; et al. Ultrahigh-energy neutrino follow-up of gravitational wave events GW150914 and GW151226 with the Pierre Auger Observatory. Phys. Rev. D 2016, 94, 122007.

29. Fukuda, S.; et al. [Pierre Auger Collaboration]. The Super-Kamiokande detector. Nucl. Instrum. Methods Phys. Res. A 2003, 501, 418-462.

30. Abe, K.; Hayato, Y.; Iida, T.; Iyogi, K.; Kameda, J.; Kishimoto, Y.; Koshio, Y.; Marti, L.; Miura, M.; Moriyama, S.; et al. Calibration of the Super-Kamiokande detector. Nucl. Instrum. Methods Phys. Res. A 2014, 737, 253-272. [CrossRef]

31. Kume, H.; Sawaki, S.; Ito, M.; Arisaka, K.; Nishimura, A.; Suzuki, A.; Kajita, T.; Nishimura, A.; Suzuki, A. 20 inch diameter photomultiplier. Nucl. Instrum. Methods Phys. Res. A 1983, 205, 443-449. [CrossRef]

32. Tanimori, T.; Ikeda, H.; Mori, M.; Kihara, K.; Kitagawa, H.; Haren, Y. Design and performance of semi-custom analog IC including two TACs and two current integrators for 'Super-Kamiokande'. IEEE Trans. Nucl. Sci. 1989, 36, 497-501. [CrossRef] 
33. Ikeda, H.; Ikeda, M.; Inaba, S.; Takasaki, F. Monolithic shaper amplifier for multianode PMT readout. Nucl. Instrum. Methods Phys. Res. A 1990, 292, 439-444. [CrossRef]

34. Nishino, H.; Awai, K.; Hayato, Y.; Nakayama, S.; Okumura, K.; Shiozawa, M.; Takeda, A.; Ishikawa, K.; Minegishi, A.; Arai, Y. High-speed charge-to-time converter ASIC for the Super-Kamiokande detector. Nucl. Instrum. Methods Phys. Res. A 2009, 610, 710-717. [CrossRef]

35. Yamada, S.; Awai, K.; Hayato, Y.; Kaneyuki, K.; Kouzuma, Y.; Nakayama, S.; Nishino, H.; Okumura, K.; Obayashi, Y.; Shimizu, Y.; et al. Commissioning of the New Electronics and Online System for the Super-Kamiokande Experiment. IEEE Trans. Nucl. Sci. 2010, 57, 428-432. [CrossRef]

36. Abe, K.; et al. [Super-Kamiokande Collaboration]. Solar neutrino measurements in Super-Kamiokande-IV. Phys. Rev. D 2016, 94, 052010.

37. Bays, K.; et al. [Super-Kamiokande Collaboration]. Supernova relic neutrino search at Super-Kamiokande. Phys. Rev. D 2012, 85, 052007.

38. Nakano, Y. Radon background study in Super-Kamiokande. J. Phys. Conf. Ser. 2017, 888, 012191.

39. Zhang, Y.; et al. [Super-Kamiokande Collaboration]. First measurement of radioactive isotope production through cosmic-ray muon spallation in Super-Kamiokande IV. Phys. Rev. D 2016, 93, 012004.

40. Richard, E.; et al. [Super-Kamiokande Collaboration]. Measurements of the atmospheric neutrino flux by Super-Kamiokande: Energy spectra, geomagnetic effects, and solar modulation. Phys. Rev. D 2016, 94, 052001.

41. Ashie, Y.; et al. [Super-Kamiokande Collaboration]. Measurement of atmospheric neutrino oscillation parameters by Super-Kamiokande I. Phys. Rev. D 2005, 71, 112005. [CrossRef]

42. Connaughton, V.; Burns, E.; Goldstein, A.; Blackburn, L.; Briggs, M.S.; Zhang, B.B.; Camp, J.; Christensen, N.; Hui, C.M.; Jenke, P.; et al. Fermi GBM Observations of LIGO Gravitational Wave event GW150914. Astrophys. J. Lett. 2016, 826, L6. [CrossRef]

43. Eichler, D., Livio, M., Piran, T.; Schramm, D.N. Nucleosynthesis, neutrino bursts and $\gamma$-rays from coalescing neutron stars. Nature 1989, 340, 126-128. [CrossRef]

44. Woosley, S.E. Gamma-ray bursts from stellar mass accretion disks around black holes. Astrophys. J. 1993, 405, 273-277. [CrossRef]

45. Surman, R.; McLaughlin, G.C.; Ruffert, M.; Hix, W.R. r-Process Nucleosynthesis in Hot Accretion Disk Flows from Black Hole-Neutron Star Mergers. Astrophys. J. Lett. 2008, 679, L117-L120. [CrossRef]

46. Caballero, O.L.; Zielinski, T.; McLaughlin, G.C; Surman, R. Black hole spin influence on accretion disk neutrino detection. Phys. Rev. D 2016, 93, 123015. [CrossRef]

47. Strumia, A; Vissani, F. Precise quasielastic neutrino/nucleon cross-section. Phys. Lett. B 2003, 564, $42-54$.

48. Nakahata, M.; Fukuda, Y.; Hayakawa, T.; Ichihara, E.; Inoue, K.; Ishihara, K.; Ishino, H.; Itow, Y.; Kajita, T.; Kameda, J.; et al. Calibration of Super-Kamiokande using an electron LINAC. Nucl. Instrum. Methods Phys. Res. A 1999, 421, 113-129. [CrossRef]

49. Swanson, M.E.; Abe, K.; Hosaka, J.; Iida, T.; Ishihara, K.; Kameda, J.; Koshio, Y.; Minamino, A.; Mitsuda, C.; Miura, M.; et al. Search for Diffuse Astrophysical Neutrino Flux Using Ultra-High-Energy Upward-going Muons in Super-Kamiokande I. Astrophys. J. 2006, 652, 206-215. [CrossRef]

50. Thrane, E.; Abe, K.; Hayato, Y.; Iida, T.; Ikeda, M.; Kameda, J.; Kobayashi, K.; Koshio, Y.; Miura, M.; Moriyama, S.; et al. Search for Neutrinos from GRB 080319B at Super-Kamiokande. Astrophys. J. 2009, 697, 730-734. [CrossRef]

51. Hayato, Y. A neutrino interaction simulation program library NEUT. Acta Phys. Pol. B 2009, 40, $2477-2489$.

52. Gaisser Thomas, K.; Halzen Francis; Stanev Todor. Particle astrophysics with high energy neutrinos. Phys. Rep. 1995, 258, 173-236. [CrossRef]

53. Yang, Y.-Z.; Ling, J.-J.; Wang, W.; Cheng, Z.-K. A global analysis searching for neutrinos associated with black hole merger gravitational wave events. Res. Astron. Astrophys. 2018, 18, 132. [CrossRef]

54. Popham, R.; Woosley, S.E.; Fryer, C. Hyperaccreting Black Holes and Gamma-Ray Bursts. Astrophys. J. 1999, 518, 356-374. [CrossRef]

55. Metzger, B.D; Berger E. What is the Most Promising Electromagnetic Counterpart of a Neutron Star Binary Merger? Astrophys. J. 2012, 746, 48. [CrossRef]

56. Sekiguchi, Y.; Kiuchi, K.; Kyutoku, K.; Shibata, M. Gravitational Waves and Neutrino Emission from the Merger of Binary Neutron Stars. Phys. Rev. Lett. 2011, 107, 051102. [CrossRef] [PubMed] 
57. Kyutoku, K.; Kashiyama, K. Detectability of thermal neutrinos from binary neutron-star mergers and implications for neutrino physics. Phys. Rev. D 2018, 97, 103001. [CrossRef]

58. Dessart, L.; Ott, C.D.; Burrows, A.; Rosswog, S.; Livne, E. Neutrino signatures and the neutrino-driven wind in Binary Neutron Star Mergers. Astrophys. J. 2009, 690, 1681-1705. [CrossRef]

59. Abbott, B.P.; et al. [LIGO Scientific Collaboration and Virgo Collaboration]. Estimating the Contribution of Dynamical Ejecta in the Kilonova Associated with GW170817. Astrophys. J. Lett. 2017, 850, L39. [CrossRef]

60. Baret, B.; Bartos, I.; Bouhou, B.; Corsi, A.; Di Palma, I.; Donzaud, C.; Van Elewyck, V.; Finley, C.; Jones, G.; Kouchner, A.; et al. Bounding the time delay between high-energy neutrinos and gravitational-wave transients from gamma-ray bursts. Astropart. Phys. 2011, 35, 1-7. [CrossRef]

61. Kimura, S.S.; Murase, K.; Meszaros, P.; Kiuchi, K. High-energy Neutrino Emission from Short Gamma-Ray Bursts: Prospects for Coincident Detection with Gravitational Waves. Astrophys. J. Lett. 2017, 848, L4. [CrossRef]

62. Biehl, D.; Heinze, J.; Winter, W. Expected neutrino fluence from short Gamma-Ray Burst 170817A and off-axis angle constraints . Mon. Not. R. Astron. Soc. 2018, 476, 1191-1197. [CrossRef]

63. Fang, K.; Metzger Brian, D. High-energy Neutrinos from Millisecond Magnetars Formed from the Merger of Binary Neutron Stars. Astrophys. J. 2017, 849, 153. [CrossRef]

64. Abe, K.; et al. [Super-Kamiokande Collaboration]. Search for an Excess of Events in the Super-Kamiokande Detector in the Directions of the Astrophysical Neutrinos Reported by the IceCube Collaboration. Astrophys. J. 2017, 850, 166. [CrossRef]

(C) 2018 by the author. Licensee MDPI, Basel, Switzerland. This article is an open access article distributed under the terms and conditions of the Creative Commons Attribution (CC BY) license (http://creativecommons.org/licenses/by/4.0/). 